\title{
Time-resolved connectome of the five-factor model of personality
}

\author{
L. Passamonti ${ }^{1,2^{*}}$, R. Riccelli ${ }^{3}$, I. Indovina ${ }^{3,4}$, A. Duggento $\mathbb{C}^{5}$, A. Terracciano $\mathbb{C}^{6}$ \& N. Toschi $\oplus^{5,7}$
}

The human brain is characterized by highly dynamic patterns of functional connectivity. However, it is unknown whether this time-variant 'connectome' is related to the individual differences in the behavioural and cognitive traits described in the five-factor model of personality. To answer this question, inter-network time-variant connectivity was computed in $n=818$ healthy people via a dynamical conditional correlation model. Next, network dynamicity was quantified throughout an ad-hoc measure ( $T$-index) and the generalizability of the multi-variate associations between personality traits and network dynamicity was assessed using a train/test split approach. Conscientiousness, reflecting enhanced cognitive and emotional control, was the sole trait linked to stationary connectivity across several circuits such as the default mode and prefronto-parietal network. The stationarity in the 'communication' across large-scale networks offers a mechanistic description of the capacity of conscientious people to 'protect' non-immediate goals against interference over-time. This study informs future research aiming at developing more realistic models of the brain dynamics mediating personality differences.

Understanding how people differ in their cognitive, emotional, and behavioural dispositions is a central theme in psychology, psychiatry, and neurology. At the intersection across these disciplines, the 'personality neuroscience' field has emerged as a new academic 'arena' where researchers strive to reveal the brain mechanisms of personality traits $^{1,2}$. So far, several neuroimaging studies have focused on linking personality measures with diverse structural and functional measures in single brain regions $s^{1,3-19}$. Others have investigated how variability in time-averaged (or static) functional connectivity relates to personality ${ }^{7,18-25}$.

However, no-one has yet assessed how the dynamic patterns of 'communications' across large-scale brain networks mediate personality differences. To offer new insights into the core neurological underpinnings of personality, we need to transition from static measures of connectivity to indices that resolve the temporal component of such connectivity patterns ${ }^{26,27}$. The most appropriate method to reveal the dynamicity (or its reverse, stationarity) in the connectivity between two 'nodes' (in this and other studies 'a node' is a 'large-scale' network) has been the subject of a fruitful debate ${ }^{28}$. Although different definitions exist ${ }^{28}$, a non-stationary connection can be quantified by the presence of frequent, long-lasting, or high excursions in the connection strength, despite the average value of the connection strength itself and the directionality of the excursion. In other words, a 'connection' between two 'nodes' (i.e., the amplitude of the correlation between two time-series) is 'dynamic' when it fluctuates frequently, for a long time, or to a large extent (or any combination of these elements). In contrast, a 'stationary' connection is one in which these connectivity patterns are minimal or infrequent.

At the cognitive level, the degree of dynamicity or stationarity in the human 'connectome' can be interpreted in different ways, depending on which 'nodes' are involved. For example, dynamic connections between networks that 'feed' external stimuli into the brain might result in 'distractibility', as, in this scenario, the rapidly changing information can 'take the lead' in driving the brain connections. Conversely, stationary connections between 'top-down' networks and 'bottom-up' sensory circuits can result in less 'distractibility', as, in this case, the incoming inputs may be 'stabilized' over-time against interference.

So far, different but partially related methods have been used to quantify the time-varying connectivity in the human brain, the most common being the 'sliding' window approach ${ }^{27-31}$. However, this method poses some limits on the temporal resolution and thus on the ability to characterize the relatively abrupt changes in the

${ }^{1}$ Institute of Bioimaging \& Molecular Physiology, National Research Council, Milano, Italy. ${ }^{2}$ Department of Clinical Neurosciences, University of Cambridge, Cambridge, UK. ${ }^{3}$ Laboratory of Neuromotor Physiology, IRCCS Santa Lucia Foundation, 00179, Rome, Italy. ${ }^{4}$ Saint Camillus International University of Health and Medical Sciences, 00131, Rome, Italy. ${ }^{5}$ Department of Biomedicine \& Prevention, University "Tor Vergata", Rome, Italy. ${ }^{6}$ Department of Geriatrics, Florida State University College of Medicine, Tallahassee, USA. ${ }^{7}$ Department of Radiology, Martinos Center for Biomedical Imaging, Boston \& Harvard medical School, Boston, USA. *email: Ip337@medschl.cam.ac.uk 


\begin{tabular}{|l|l|l|}
\hline Demographic variables & $367 / 451$ \\
\hline Gender (males/females) & $28.7 \pm 3.7[22-37]$ \\
\hline Age (years) & $743 / 73 / 2$ \\
\hline Handedness (Right/Left/Both) & $14.9 \pm 1.8[11-17]$ \\
\hline Education (years) & Hispanic/Latino & $8.6 \%$ \\
\cline { 2 - 3 } & Not Hispanic/Latino & $90.5 \%$ \\
\cline { 2 - 3 } Ethnicity (\%) & Unknown/Not Reported & $0.9 \%$ \\
\hline & & \\
\hline Personality scores (NEO-FFI) & \multicolumn{2}{|l|}{} \\
\hline Neuroticism & $16.3 \pm 7.2[0-43]$ \\
\hline Extraversion & $30.7 \pm 5.9[11-47]$ \\
\hline Openness & $28.3 \pm 6.1[12-45]$ \\
\hline Agreeableness & $32.0 \pm 5.0[13-45]$ \\
\hline Conscientiousness & $34.5 \pm 5.9[12-48]$ \\
\hline
\end{tabular}

Table 1. Age, education, and personality data are expressed as mean \pm standard deviation while the range in square brackets [] is expressed as minimum-maximum. NEO five-factors inventory questionnaire, NEO-FFI.

functional connectivity patterns that are commonly observed in resting-state functional magnetic resonance imaging (rs-fMRI) data ${ }^{29}$. Here, we employed a dynamic conditional correlation (DCC) model that has been specifically developed for rs-fMRI datasets ${ }^{32}$. The DCC is based on a point-by-point volatility model (a generalized autoregressive conditional heteroscedastic - GARCH -model) and is robust against apparent changes in correlations caused by random noise ${ }^{30}$. These features make it an attractive tool for resolving the time-variant associations in datasets with low signal-to-noise ratio such as the rs-fMRI data.

We also sought to identify the time-resolved functional connectivity patterns that related to the cognitive and behavioural dispositions described in the five-factor model (FFM) of personality ${ }^{33,34}$. The FFM traits (i.e., neuroticism, extraversion, openness, agreeableness, and conscientiousness) have been empirically derived from a large body of epidemiological and psychosocial research ${ }^{35-38}$. The FFM traits also display sufficient universality across different demographic and cultural groups and predict important outcome measures such as educational or occupational success, risk to develop dementia, and longevity ${ }^{35-38}$.

Overall, this study was exploratory in its nature as there was (to the best of our knowledge) no other study assessing the relationship between time-variant connectivity measures and personality traits. However, we had some expectations regarding a specific effect of conscientiousness on such time-resolved connectivity indices. This is because we found, in the same sample of participants used here, that static (i.e., time-averaged) 'connectomic' measures (i.e., nodal strength, local clustering, and betweenness-centrality) positively related to conscientiousness, but not to any other FFM trait ${ }^{25}$. Hence, here, we tested whether conscientiousness was the sole FFM trait linked to time-resolved connectivity measures, over and above its effect on 'static' connectivity indices.

Nevertheless, it was reasonable to hypothesize that other FFM traits could have been linked to more or less dynamic connectivity patterns (e.g., openness could have been associated to more dynamic connectivity across sensory-related circuits).

\section{Results}

Participants. All data were drawn from $n=818$ individuals from the Human Connectome Project (HCP) database, a large repository of behavioural and neuroimaging measures. The demographic and personality variables of the HCP sample are summarized in Table 1. Most participants were right-handed white Americans. Less than $10 \%$ had a Hispanic or Latino background.

Potentially confounding variables such as age, sex, years of education, handedness, and intelligence scores were included as 'nuisance' variables in the statistical models testing for the relationship between the FFM personality traits and the time-resolved connectivity patterns.

Neuroimaging findings. Independent components analysis (ICA) of large-scale networks 'nodes'. Brain networks were identified via group-ICA (dimensionality: $n=15$ ) calculated by the HCP consortium and were characterized by a series of brain regions that have been reported in earlier studies (e.g., the sensory-motor circuit, the visual circuits, the default-mode network, the left and right fronto-parietal circuits, the salience network, etc.) ${ }^{39,40}$ (Fig. 1 and Supplementary Table 1 for a list of the anatomical regions belonging to each network 'node'). Average connectivity strengths between nodes were also provided by the HCP consortium ('netmats2'). Each of these large-scale networks was considered as a 'node' in the time-resolved connectivity analyses described in the Methods section.

Dynamical connectivity results independently of personality differences. First, the time-variant connectivity was estimated via the dynamic conditional correlation (DCC) model developed for rs-fMRI datasets ${ }^{32}$. A connection-wise index of non-stationarity (“T”-index) (initially introduced by Zalesky and colleagues) was computed for each connection (Fig. 2 for examples from real data) ${ }^{27}$. The higher the " $T$ "-index, the more 'dynamic' (i.e., 'non-stationary') a connection is (see Methods section). 


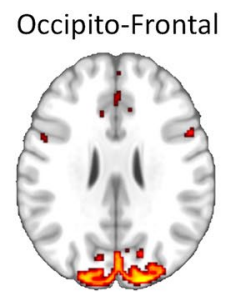

Right Fronto-Parietal
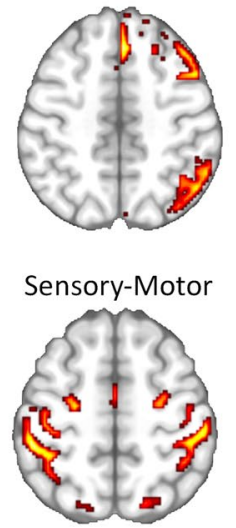
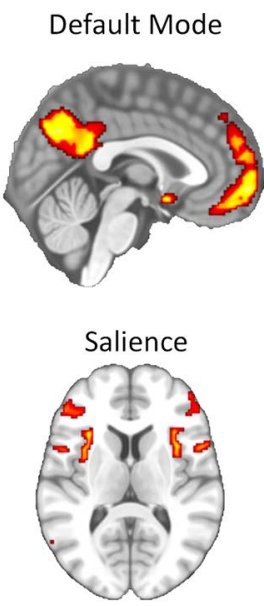

Fronto-Temporal

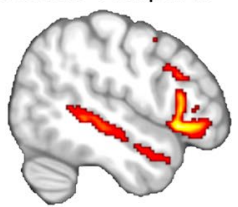

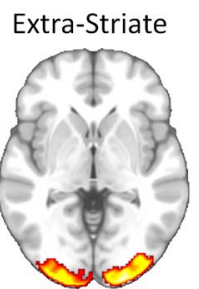

Striate

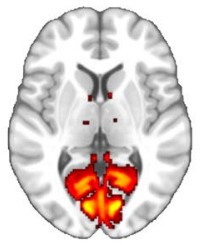

Sensory/Motor-Limbic

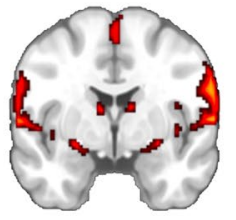

Occipito-Parietal

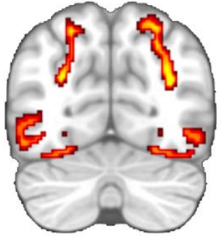

Hippocampal-Cerebellar
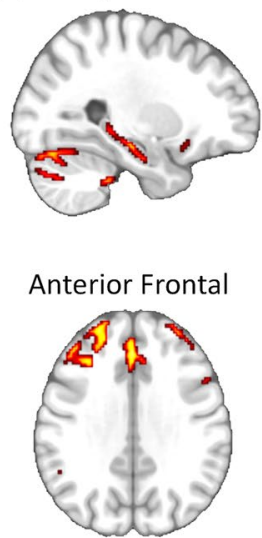

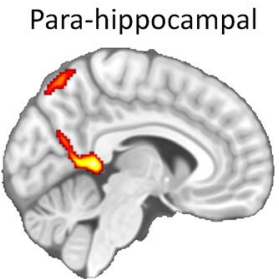

Left Fronto-Parietal
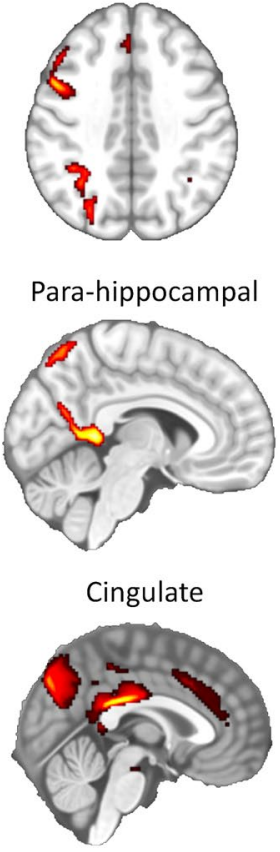

Figure 1. Resting-state 'large-scale' networks identified via independent component analysis (ICA). By using group ICA, the Human Connectome Project consortium has identified fifteen distinct brain circuits that we used to study the time-resolved connectivity patterns. The complete list of the brain region in each resting-state network is reported in Supplementary Table 1.

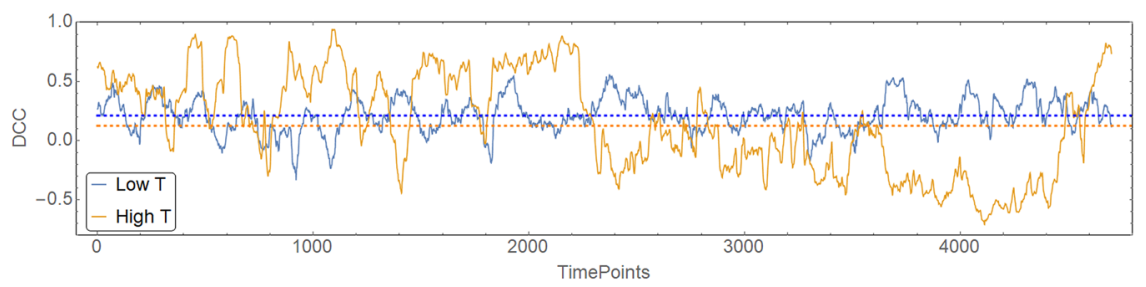

Figure 2. Examples of high- and low- dynamicity (i.e. T-index) in time-resolved connectivity. Two types of time-resolved connections are depicted, one with high-dynamicity (high " $\mathrm{T}$ "-index, light brown line) and the second with low-level of dynamicity (i.e. high stationarity) (low " $\mathrm{T}$ "-index, blue line). The dotted lines represent the median values for each connection (i.e. static/time-averaged connectivity). The more dynamic connection exhibits more, higher, and longer-lasting excursions from the overall median. DCC, dynamic conditional correlation.

Second, the dynamicity of each connection (i.e. its "T"-index) was plotted against the static strength of the same connection (obtained from the HCP consortium and calculated using partial correlation) as shown in Fig. 3.

Independently of personality differences, the connections between visuo-parietal networks (occipito-parietal/ occipito-frontal) and visual circuits (striate/extrastriate) that 'feed' external input into the brain were amongst the most dynamic (Fig. 3). Connections between occipito-frontal and sensory/motor networks and within sensory-motor circuits (i.e., sensory/motor-limbic-sensory/motor) also displayed a relatively high level of dynamicity (Fig. 3). In contrast, the connections across networks involved in cognitive controls (e.g., cingulate network, default mode network, hippocampal/para-hippocampal circuits) showed more stationary patterns of connectivity (Fig. 3).

Correlations between time-resolved indices of functional connectivity and personality traits. Next, we studied how the dynamicity of the connections across the nodes was related to individual differences in the FFM traits. To evaluate the generalizability of our findings, the initial sample of $n=818$ participants was split into two sub-samples: a 'training' set ( $75 \%$ of participants, $n=613)$ and a 'test' set ( $25 \%$ of participants, $n=205)$. In the training set, we used multi-variate regression analyses (i.e., general linear models, GLMs) with the " $\mathrm{T}$ "-index as dependent variable, to explore the associations between the dynamicity of each connection and each FFM trait while accounting for potentially confounding effects driven by the remaining FFM traits as well as other 'nuisance' 


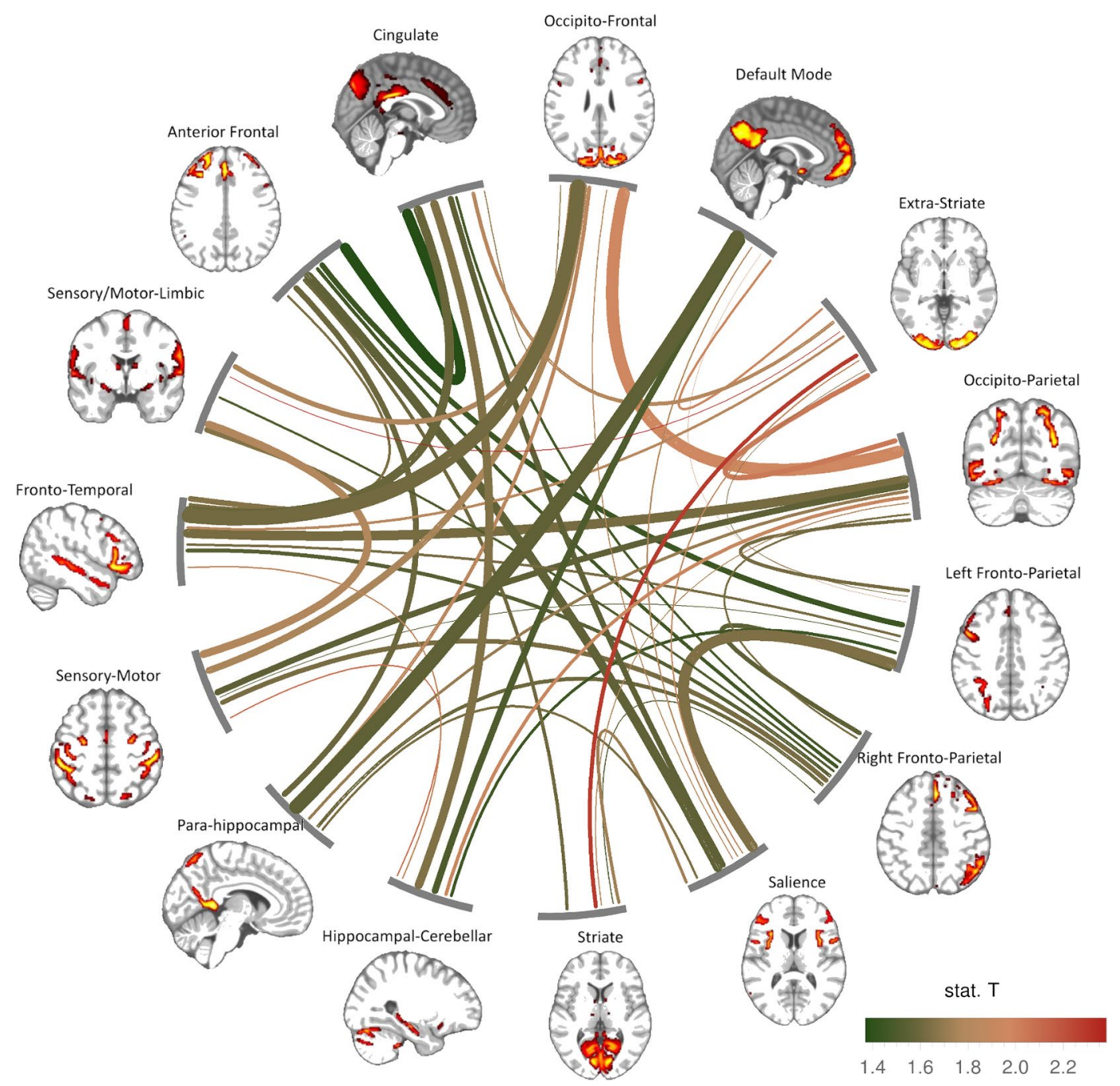

Figure 3. Dynamicity and strength of inter-network connectivity regardless of personality differences. The colour of the lines connecting each pair of 'nodes' (large scale-networks) reflects the degree of dynamicity (red: more dynamic, green: less dynamic, as assessed by the " $T$ "-index, see Methods and Fig. 2). The thickness of each line represents the strength of the connection between each couple of nodes (with thicker lines reflecting stronger connections, as assessed by the HCP consortium in the 'netmats2' version). Both strength and dynamicity are represented through median values across all subjects. Only connections associated with positive strength (i.e. partial correlation, as provided by the HCP consortium) are shown.

factors such as sex, age, education, handedness, and intelligence scores. Associations surviving a threshold of $\mathrm{P}<0.05$ (false-discovery-rate-FDR-corrected across 105 connections) were considered statistically significant. The associations discovered (i.e., the regression models estimated) in the 'training' dataset were used to assess the generalizability of our findings in the 'test' dataset (see Methods) using the relative root mean square error (RRMSE) as a criterion of merit ${ }^{41}$.

No significant associations, either positive or negative, were found between neuroticism (P's $>0.2)$, extraversion (P's $>0.6$ ), openness (P's $>0.2$ ), and agreeableness (P's $>0.9$ ) scores and the dynamicity (“T”-index) of any connection. In contrast, negative associations were found between conscientiousness scores and the " $T$ "-index across a series of networks including prefronto-parietal and prefronto-temporal networks, the default mode network, cingulate circuits, sensory-motor and limbic networks as well as posterior occipito-parietal circuits (P's $<0.05$, FDR) (Table 2, Fig. 4). Of note, we achieved good generalizability of our findings in all cases in which we found a significant effect in the GLM analyses (Table 2, Fig. 4) (median RRMSE =0.14, maximum RRMSE $=0.20)^{41}$.

To exclude that our main findings were not confounded by personality-driven differences in head movement during scanning, we also tested for associations between the average root-mean-square (RMS) displacement across runs and conscientiousness scores. No significant correlation was found between RMS and conscientiousness (Pearson's $\mathrm{R}=0.04, \mathrm{P}=0.24$ ). 


\begin{tabular}{|l|l|l|l|l|}
\hline ICA node\# 1 & ICA node\#2 & $\begin{array}{l}\text { P value } \\
\text { (FDR) }\end{array}$ & $\begin{array}{l}\text { Effect } \\
\text { size }\end{array}$ & RRMSE \\
\hline Extra-striate & Cingulate & 0.012 & 0.154 & 0.167 \\
\hline Sensory/Motor-Limbic & Cingulate & 0.012 & 0.157 & 0.188 \\
\hline Occipito-Frontal & Extra-striate & 0.041 & 0.123 & 0.180 \\
\hline Occipito-Frontal & Fronto-Temporal & 0.041 & 0.125 & 0.168 \\
\hline Default-Mode & Right-Fronto-Parietal & 0.041 & 0.125 & 0.186 \\
\hline Default-Mode & Sensory/Motor-Limbic & 0.041 & 0.122 & 0.185 \\
\hline Extra-striate & Anterior Frontal & 0.041 & 0.131 & 0.154 \\
\hline Hippocampal-Cerebellar & Cingulate & 0.041 & 0.130 & 0.189 \\
\hline Sensory-Motor & Sensory/Motor-Limbic & 0.041 & 0.127 & 0.176 \\
\hline Occipito-Frontal & Striate & 0.046 & 0.114 & 0.177 \\
\hline Occipito-Frontal & Sensory-Motor & 0.046 & 0.118 & 0.145 \\
\hline Extra-striate & Occipito-Parietal & 0.046 & 0.119 & 0.173 \\
\hline Extra-striate & Hippocampal-Cerebellar & 0.046 & 0.114 & 0.175 \\
\hline Occipito-Parietal & Right-Fronto-Parietal & 0.046 & 0.116 & 0.195 \\
\hline Occipito-Parietal & Sensory-Motor & 0.046 & 0.114 & 0.149 \\
\hline Default-Mode & Striate & 0.046 & 0.113 & 0.142 \\
\hline Fronto-Temporal & Sensory/Motor-Limbic & 0.049 & 0.111 & 0.189 \\
\hline
\end{tabular}

Table 2. Connections between pairs of large-scale networks 'nodes' (first and second column) in which negative associations between conscientiousness scores and the " $\mathrm{T}$ "-index of dynamicity were found while accounting for the other personality traits as well as variability in sex, age, handedness, years of education, and intelligence scores (FDR: false discovery rate correction for multiple comparisons across 105 connections). Effect sizes were estimated using partial correlations between the " $T$ "-index and all independent variables in the model, ensuring full overlap between the estimated model coefficients and corresponding normalized effect sizes. Relative root mean square error (RRMSE) resulting from employing the estimated models on the test set is also reported. Model accuracy is considered excellent when RRMSE $<0.1$, good when $0.1<$ RRMSE $<0.2$, fair when $0.2<$ RRMSE $<0.3$, and poor if RRMSE $>0.3^{41}$.

\section{Discussion}

We provide new evidence that people scoring high in conscientiousness display more stationary connectivity patterns across large-scale networks that have been implicated in cognitive control (i.e., prefronto-parietal, prefronto-temporal, default mode, anterior frontal, and cingulate networks), visuo-spatial and visuo-motor skills (striate, extra-striate, occipito-parietal, and occipito-frontal networks) and sensory/motor and emotional functions (sensory-motor and limbic networks). The relationship between conscientiousness and stationary connections was obtained using multi-variate statistical models that controlled for the remaining FFM traits (i.e., neuroticism, extraversion, openness, and agreeableness) and for variability in potentially confounding factors such as age, sex, handedness, education, and intelligence. Of note, the generalizability of our findings was supported by a 'training' and 'test' split validation approach.

We also found that, independently of personality differences, the connections across networks 'feeding' external inputs into the brain (occipito-parietal, occipito-frontal, striate, and extra-striate) and those regulating the motor output (i.e., sensory/motor-limbic-sensory/motor) displayed the highest levels of dynamicity. In contrast, the connections across networks involved in cognitive control (e.g., cingulate network, default mode network, anterior frontal circuits) showed more stationary connectivity patterns.

These data suggest that temporally resolved 'connectomic' indices are reliable markers that can be used as indicators of the cognitive and behavioural style across different people; for example, the ability of conscientious persons to efficiently pursue specific goals and maintain them consistent over time ${ }^{42-45}$. Furthermore, the current results support and extend our recent data showing that people scoring high in conscientiousness display increased nodal strength, local clustering, and betweenness-centrality across similar circuits ${ }^{25}$. Together, these findings mechanistically describe why conscientious people display a cognitive style that is resistant to the disruptive interference of incoming stimuli, which are often emotional in nature ${ }^{46}$.

This interpretation is in keeping with a theoretical framework positing that conscientiousness would contribute to the behavioural construct of 'stability', a meta-trait that would have evolved from the necessity to prevent the disruption of goals by interfering stimuli ${ }^{46}$. However, no significant results were obtained for the other 'stability' traits (i.e., low neuroticism and high agreeableness) which indicates that such effects do not necessarily extend to the meta-trait of 'stability'. On the other hand, the temporal stationarity of the connectivity patterns in conscientious people can be interpreted in the context of previous studies showing that, across different samples and age-groups, conscientious people tend to display more 'constant' behavioural traits over time ${ }^{47-51}$.

The association between conscientiousness and stationary connectivity patterns was localized across networks that have been implicated in 'top-down' cognitive control, 'bottom-up' input-processing functions, and action generation. In particular, the fact that stationary interactions were found between prefronto-parietal and occipito-parietal/extra-striate circuits as well as between the default mode network and the sensory-motor, prefronto-parietal, and the visual striate networks is consistent with the notion that conscientious people maintain well the focus on non-immediate goals ${ }^{1,52,53}$. 


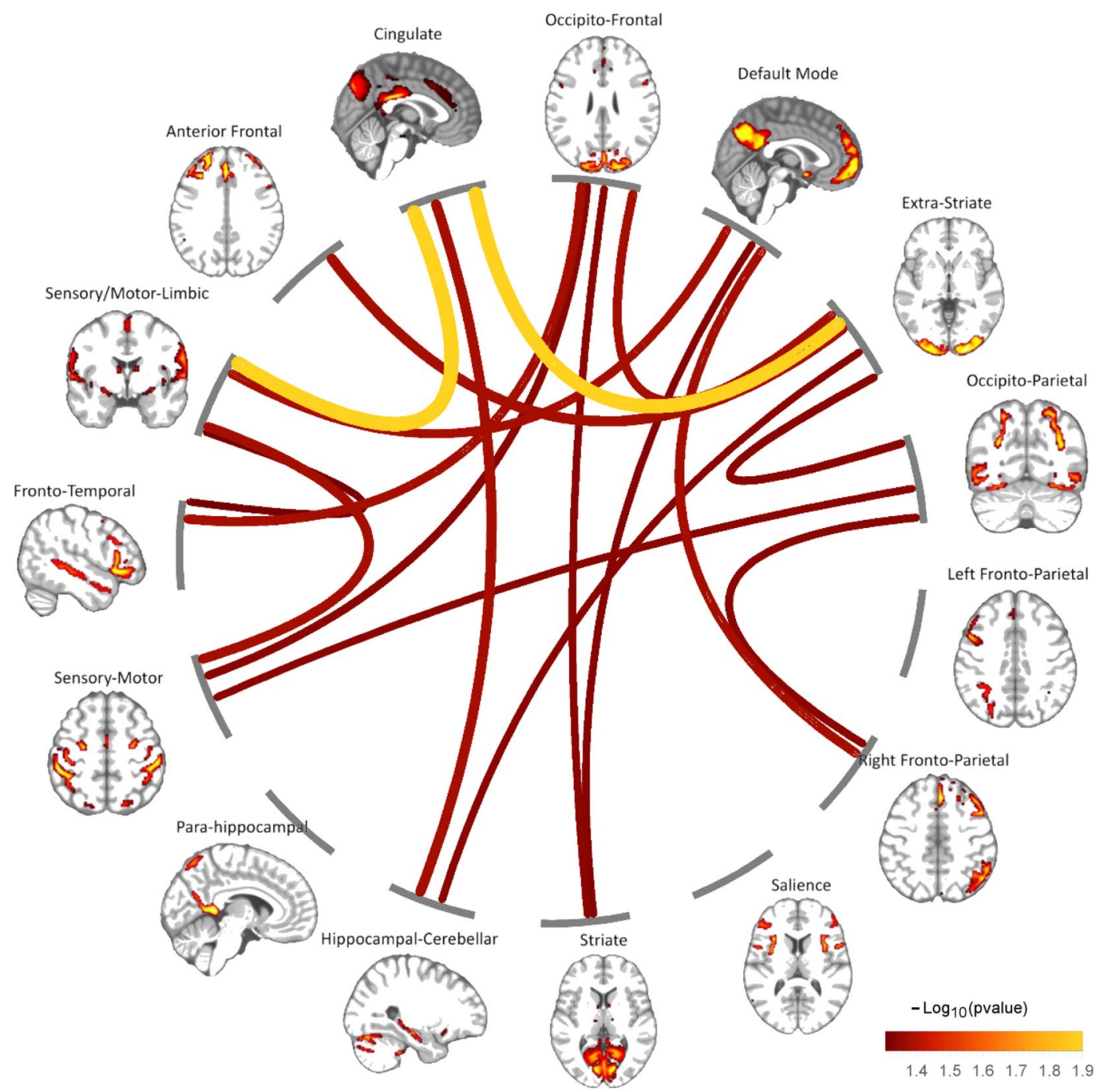

Figure 4. Conscientiousness was the only personality trait that was linked to stationary connectivity patterns across large-scale networks after accounting for potentially confounding factors such as the remaining personality traits, age, sex, years of education, handedness, and intelligence. The colour of the lines connecting each pair of 'nodes' (large scale-networks) represents the statistical significance $\left(-\log _{10}-\mathrm{p}\right.$-value, yellow colour representing lower p-values: e.g. $-\log _{10}(0.05)=1.30$ and $\left.-\log _{10}(0.01)=2\right)$ of the negative associations found between conscientiousness scores and the " $\mathrm{T}$ "-index of each connection. The thickness of each line represents the effect sizes for each connection with thicker lines reflecting higher effect sizes. All associations shown survive a threshold of $\mathrm{P}<0.05$, correction for multiple comparisons using false discovery rate across all possible 105 connections (see Table 2).

At the same time, the stationary patterns of functional interactions between the cingulate cortices and sensory-motor limbic networks may help explaining why conscientious people successfully adjust their behavioural responses to different environmental contexts; for example, by delaying immediate gratification ${ }^{1,52,53}$. Stationarity in the patterns of 'communication' between memory circuits (i.e., hippocampal/cingulate networks) can also mediate a good performance during prospective memory tasks that require retrieval of complex sequences of planned actions ${ }^{54-56}$.

Strengths and limitations. The use of a large sample of participants $(n=818)$, state-of art analytical pipelines (i.e., DCC model), and the inclusion of a training/test-set for assessing the generalizability of our findings are the main methodological advantages of this study.

The fact that conscientiousness was the sole personality trait related to time-variant connectivity measures is consistent with our recent data showing that conscientiousness was the only FFM factor linked to heightened static 'connectomic' metrics such as nodal strength, local clustering, and betweenness-centrality ${ }^{25}$. Nevertheless, our earlier and current studies do not necessarily imply that other FFM traits do not have any brain functional correlate as there can be several reasons for null results including: (i) type II errors; (ii) non-linear relationships between FFM traits and time-variant metrics, and (iii) the fact that correlations between time-variant functional 
measures and personality traits might exist but could only be revealed by more narrow measures of personality (i.e., facets within each of the five factors) or other (e.g., task-related) functional measures.

Another issue regards the level of anatomical 'granularity' of the brain networks that is required to reveal the relationship between the time-resolved patterns of connectivity and personality differences. In other words, one could hypothesize that it is: 1) the interaction between large-scale brain networks that relates to the dynamicity or stability of functional connections, and/or 2) the more fine-grained interplay between individual areas within a network (e.g., anterior and posterior components of the default mode network) that is linked to the cognitive/ behavioural differences described by the FFM of personality. Although either possibilities are equally plausible, non-mutually exclusive, and potentially interesting to study, we assumed here that a large-scale network approach would have retained a sufficient level of anatomical detail to reveal the relationship between time-variant connectivity and differences in the FFM traits. The use of large-scale circuits ( $n=15$ ICA-derived networks) also enabled us to compare the findings from this study to those reported in the previous one ${ }^{25}$.

Finally, in this study, as in many others using rs-fMRI, people's performance during fixation (i.e., eye tracking data) was not monitored. However, eye tracking data in a limited group of participants $(n=132)$, for which the 3 Tesla MRI data were also available, was subsequently collected during MRI scanning at ultra-high field (7 Tesla). Hence, we assessed the potential impact of eye movements during scanning on our main outcome measure (T-index) and found that it was negligible (see supplementary material). Although this result does not exclude $a$ priori whether participants' ability to fixate affected our current findings (as the session with the eye tracking data at $7 \mathrm{~T}$ and the sessions at $3 \mathrm{~T}$ were on separate days); it showed in principle that the calculation of the $\mathrm{T}$-index is not influenced by eye movements during rs-fMRI.

The potential confound of eye movements or closure during scanning can also be partially mitigated via reliable procedures that correct for head movements that are typically associated with fatigue or lapses in concentration during scanning. In our study, the noise related to head movements was removed via the HCP-specific ICA-FIX automated algorithm (which has $\sim 99 \%$ sensitivity and specificity in de-noising HCP data ${ }^{57-59}$ ). Furthermore, we did not find any relationship between conscientiousness and head displacement during scanning which suggests negligible effects of personality differences on mediating compliance during rs-fMRI scanning. However, as before, we cannot completely exclude $a$ priori any interaction between the de-noising procedures and the correct preservation of the personality specific characteristics in the rs-fMRI signal.

\section{Conclusions}

We found a negative association between conscientiousness scores and the dynamicity of the time-resolved functional connectivity patterns. Together with our recent results showing enhanced connectivity strength, local clustering, and betweenness centrality in cognitive networks in relation to conscientiousness, the current findings provide new mechanistic insights for the empirical observation that conscientious people are superb in maintaining long-term plans consistent over time.

\section{Methods}

Personality assessment. The FFM personality traits were assessed with the NEO five-factors inventory (NEO-FFI), which is composed by 60 items, 12 per each of the five factors ${ }^{33,60}$. For each item, participants reported their level of agreement on a 5-points Likert scale, from strongly disagree to strongly agree. This NEO instrument has been previously validated in the US and several other countries ${ }^{35}$. The recently discovered bug in the scoring of $\mathrm{HCP}$ agreeableness data was corrected prior to any further processing (personal communication on HCP mailing list on 03/09/2018 20:48 CEST). All data used in the present study are available for download from the Human Connectome Project (www.humanconnectome.org). Users must agree to data use terms for the HCP before being allowed access to the data and ConnectomeDB, details are provided at (https://www.humanconnectome.org/study/hcp-young-adult/data-use-terms). The HCP has implemented a two-tiered plan for data sharing, with different provisions for handling Open Access data and Restricted data (e.g., data related to family structure, age by year, handedness, etc). This study was carried out in compliance with the HCP restricted use data terms (https://www.humanconnectome.org/study/hcp-young-adult/document/wu-minn-hcp-consortium-restricted-data-use-terms). In particular, with reference to point 6 in the aforementioned document, local regulations at one of the PIs' site (University of Rome Tor Vergata) do not require separate or individual ethics committee submission and/or approval.

MRI scanning protocol and pre-processing. Rs-fMRI data were acquired using a 3T scanner (Siemens AG, Erlangen, Germany) ${ }^{61}$. Four runs of approximately 14 minutes and 24 seconds each were obtained. Subjects lied within the scanner with open eyes while fixating a bright central cross projected on a dark background. Oblique axial acquisitions were alternated between phase encoding in a right-to-left direction in one run and phase encoding in a left-to-right direction in the other run. Gradient-echo echo-planar imaging used the following parameters: $\mathrm{TR}=720 \mathrm{~ms}, \mathrm{TE}=33.1 \mathrm{~ms}$, flip angle $=52^{\circ}, \mathrm{FOV}=208 \times 180 \mathrm{~mm}$, Matrix $104 \times 90$, Slice thickness $=2.0 \mathrm{~mm} ; 72$ slices; $2.0 \mathrm{~mm}$ isotropic voxels, Multiband factor $=8$, Echo spacing $=0.58 \mathrm{~ms}, \mathrm{BW}=2290 \mathrm{~Hz} /$ Px. This resulted in 4,800 rs-fMRI volumes in total per subject, subdivided in 4 runs of 1,200 volumes each. Structural (T1-weighted) images and field maps were also acquired to aid data pre-processing. Within the HCP consortium, each 1,200 brain volumes run of each subject's rsfMRI data was minimally pre-processed according to the latest version (3.1) of the HCP pipeline ${ }^{62}$.

Each dataset was temporally de-meaned and had variance normalization applied according to Beckmann and colleagues \{Beckmann, 2004 \#280\}. Group-principal component analysis (PCA) output was generated by MIGP (MELODIC's Incremental Group-PCA) from $n=818$ participants. This comprises the top 4,500 weighted spatial eigenvectors from a group-averaged $\mathrm{PCA}^{63}$. The MIGP output was then fed into group-independent component analysis (ICA) using FSL's MELODIC tool ${ }^{64}$, applying spatial-ICA at dimensionality of 15 . Successively, the ICA 


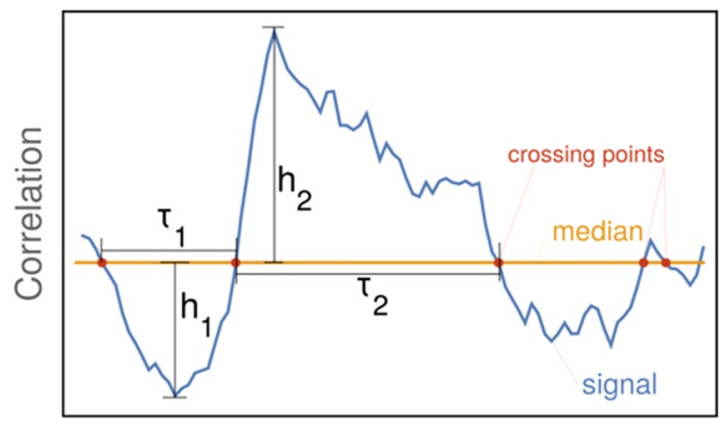

Time

Figure 5. Example of excursions around the median value of a DCC estimate. Excursion length $\tau_{1}$ and $\tau_{2}$ and excursion magnitudes $\mathrm{h}_{1}$ and $\mathrm{h}_{2}$ are shown.

maps (dimensionality: 15) were dual-regressed into each subject's $4 \mathrm{D}$ dataset to give a set of 15 time-courses of 4,800 time points per subject. Further details regarding data acquisition and pre-processing can be found in the HCP S900 Release reference manual available at https://www.humanconnectome.org/. Node maps (Fig. 1) as well as node- and subject-specific time-series were obtained from the HCP database for further processing. From the same database we also obtained connectivity matrices computed through partial correlation ('netmats2', https:// db.humanconnectome.org/data/projects/HCP_1200) to be included in our analysis.

Estimation of time-variant functional connectivity. Dynamic connectivity estimation. To estimate DCC, we follow the methods outlined and the code distributed with the original publication ${ }^{32}$. GARCH volatility models assume that the conditional variance at time $t$ is a linear combination of the past values of the conditional variance and of the past values of the squared process itself. The minimal univariate GARCH model takes the form:

$$
y_{t}=\sigma_{t} \varepsilon_{t}
$$

where $\varepsilon_{t}$ is a normal variable and the conditional variance $\sigma_{t}$ is a function of previous time-step of the signal and of the variance itself:

$$
\sigma_{t}^{2}=\omega+\alpha y_{t-1}^{2}+\beta \sigma_{t-1}^{2}
$$

where $\omega>0, \alpha \geq 0, \beta \geq 0$ and $\alpha+\beta<1$. The algorithm employed in this paper for any two fMRI time-series (i.e., the DCC algorithm $)^{65}$ consists of the following three steps: i) given that GARCH models account for volatility around a mean, all signals are de-trended using an autoregressive integrated moving average (ARIMA) model; ii) univariate GARCH models are fitted to each of the two time-series and standardized residuals are computed, and iii) an exponentially weighted moving average (EWMA)-type method is applied to the standardized residuals to compute a non-normalized version of the time-varying correlation matrix $\mathbf{R}_{t}$, which is rescaled-see equations 19-24 in Lindquist et al. ${ }^{32}$ - to obtain a conditional covariance. This procedure allows the estimation of time-varying, dynamical conditional covariance between the two components of $y_{t}$ which, in the case of two rs-fMRI time-series, can be employed as an estimate of dynamical connectivity, i.e. the DCC ${ }^{32}$ from the off-diagonal elements of the conditional covariance matric. The DCC which is an estimate of the association between the two time-series (i.e. connectivity) at the same temporal resolution as the original signals. All parameters are estimated using maximum likelihood methods.

After DCC estimation, for each DCC time-series (i.e., for each time-varying connection) we computed a non-stationarity index, term " $T$ "-index" as explained in the following paragraph.

T-index for quantifying non-stationarity. The non-stationarity T-index used here to evaluate the non-stationarity of time-varying connectivity was inspired by the T-index originally developed by Zalesky et al. ${ }^{27}$. Such T-index is based on the rationale that the non-stationarity property of a time-resolved correlation depends on large and prolonged excursions from the median connectivity value over-time. The larger and/or more prolonged such excursions in connectivity are, the more "dynamical" (i.e., non-stationary) the connection is considered to be. The T-index is defined and calculated as follows: (see also supplementary information in Zalesky et al.) ${ }^{27}:$ (a) an excursion is defined by two consecutive crossing points around the median value (see Fig. 5); (b) the excursion's length $\tau$ is defined by the time difference between two consecutive crossing points; (c) the excursion's magnitude $h$ is defined as the absolute difference between the highest or lowest time series correlation and its median value, within the context of each specific excursion. Figure 5 graphically summarizes these definitions.

Formally, the T-index of dynamicity is defined as follow:

$$
\text { T-index }=\frac{1}{N} \sum_{i}^{N} h_{i} \tau_{i} .
$$

where $\mathrm{N}$ is the number of excursions. 

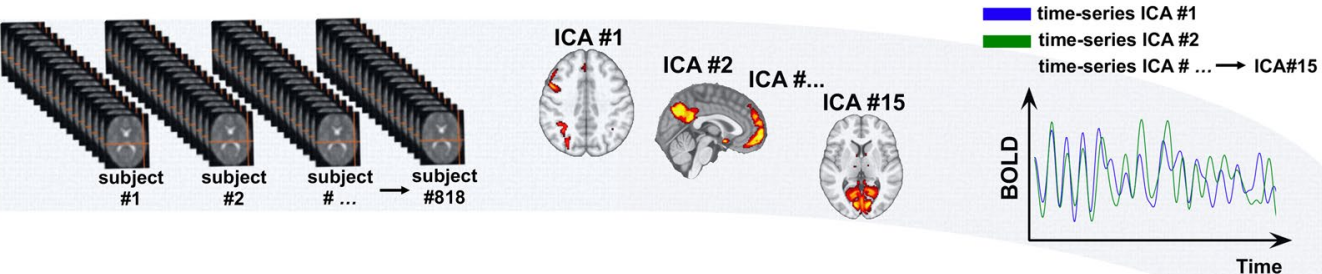

Stationarity Measure Dynamic Functional Connectivity Matrices Dynamic Conditional Correlation
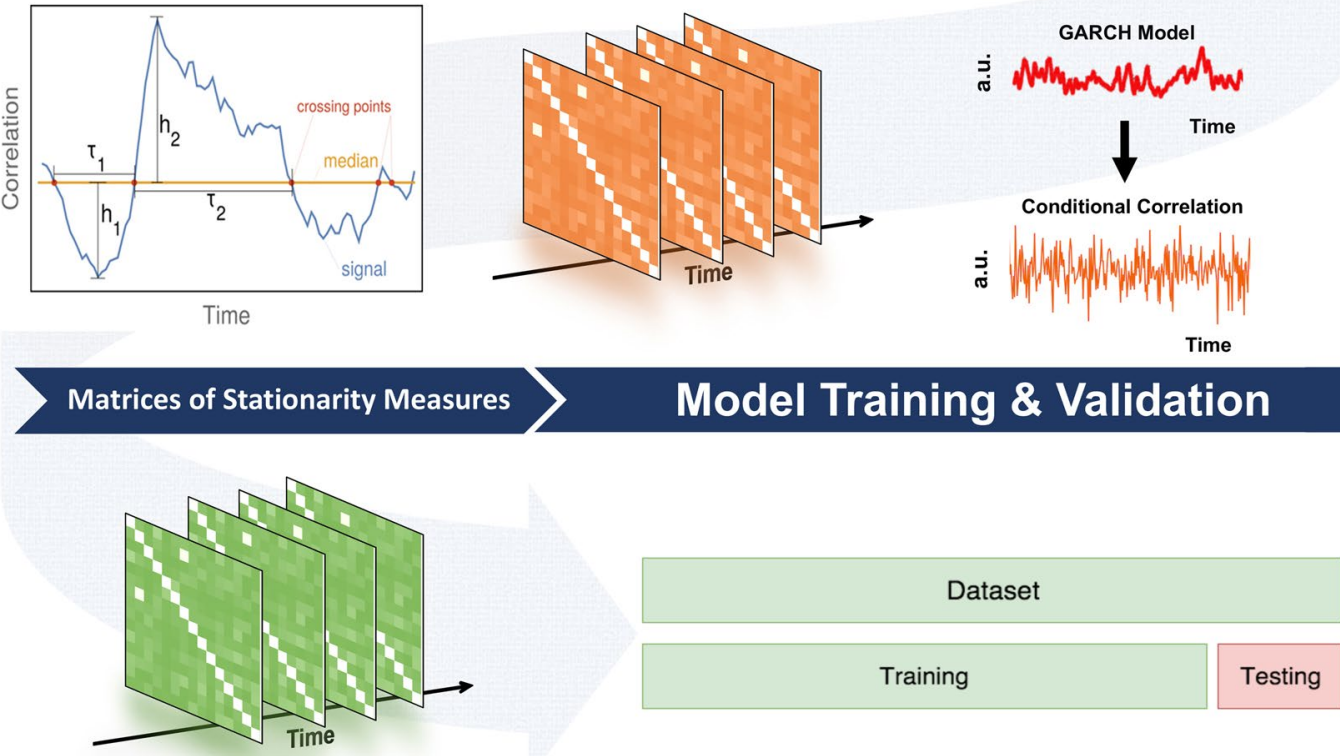

Figure 6. Image analysis workflow. The resting-state functional magnetic imaging (fMRI) data were initially pre-processed and next a set of 15 separate brain circuits were extracted via independent components analysis (ICA). Next, time-series from each ICA brain circuit were obtained from each individual and fed into dynamic conditional correlation (DCC) models. This led to $15 \times 15$ time-variant functional connectivity matrices at the single-subject level that were then used to estimate non-stationarity " $\mathrm{T}$ "-index as in Zalesky et al. ${ }^{27}$. Finally, these " $\mathrm{T}$ "-index values were analyzed in conjunction with the traits of the five-factor-model of personality at the group level. The generalization ability of the model was evaluated using a using a train/test split approach (see the statistical analyses section of the methods for further information).

To exclude potential confounding effects driven by differences in scan sessions (1st and 2 nd half of the time-series), we conducted a series of additional analyses reported in supplementary material.

Moreover, to assess the impact of eye movements during scanning on the quantification of the T-index, we performed further analyses in a sub-group of people $(n=132)$ for which eye-tracking data were available during subsequent scanning at ultra-high-field (7 Tesla) (see supplementary material). Eye-tracking data (along with all other HCP data) are available as part of the $7 \mathrm{~T}$ data release at db.humanconnectome.org.

Statistical analyses. Generation of train-test samples. To test the associations between non-stationarity measures and personality traits, as well as to evaluate the generalizability of our findings, the initial sample of $n=818$ participants was split into two matched sub-samples: a 'training' set ( $75 \%$ of participants, $n=613)$ and a 'test' set $(25 \%$ of participants, $n=205)$. The matched train/test split was generated by random sampling (with replacement) and subsequent comparison between the resulting train and test sets through nonparametric Mann-Whitney-U tests until no significant differences in the median "T"-index (across subjects) for any 105 possible connections was found. This ensured that the train and test set had comparable dependent variable distributions across all possible connections.

Inference of associations between non-stationarity and personality. The 'training' sample was used to estimate the association between the " $\mathrm{T}$ "-index (i.e., non-stationarity measure) described above and the FFM personality traits. Specifically, general linear models (GLMs), including each of the FFM traits as well as age, sex, handedness, education, and intelligence scores as covariates of no interest, were fitted to each connection (dependent variable: non-stationarity index “ $T$ ”). The resulting P-values were corrected for multiple comparisons across all possible 
105 connections using a false discovery rate (FDR) procedure. Associations surviving a threshold of $\mathrm{P}<0.05$ (FDR-corrected) were considered statistically significant. The results were a set of FDR corrected P-values as well as multivariate regression coefficients for each connection. For each regression (i.e., for each connection), effect sizes were estimated using the absolute values of partial correlations between the " $T$ "-index and all independent variables in the model. As partial correlation is defined through linear regression, this ensured full overlap between the estimated model coefficients and corresponding normalized effect sizes.

Generalizability to unseen test set. Finally, the 'test' sample (i.e., in an unseen group of subjects to which the model was completely 'agnostic') was employed to assess the generalizability of the multivariate models fitted on the 'training' set. To this end, the GLMs fitted in the training set were used to estimate the " $T$ "-indices in the 'test' sample using the demographic variables and personality scores of the 'test' sample as inputs (i.e., the rs-fMRI data of the 'train' sample was not employed in this procedure). The similarity between 'real' "T"-indices (i.e., computed using rs-fMRI data from the 'test' sample) and 'estimated' time-variant indices (i.e., predicted using the GLM coefficients fitted on 'training' data only) was assessed through the relative root mean square error (RRMSE), a normalized version of the root mean squared error which is often used as a measure of the differences between predicted and observed values. Model accuracy can be considered excellent when RRMSE $<0.1$, good when $0.1<$ RRMSE $<0.2$, fair when $0.2<$ RRMSE $<0.3$, and poor if RRMSE $>0.3^{41}$. This ensured comparability of generalization capability across the personality traits. The image analysis pipeline is summarized in Fig. 6.

Received: 19 March 2019; Accepted: 2 October 2019;

Published online: 21 October 2019

\section{References}

1. DeYoung, C. G. Personality Neuroscience and the Biology of Traits. Social and Personality Psychology Compass 4, 1165-1180 (2010).

2. Corr, P. Understanding biological psychology. (Blackwell, 2006).

3. Canli, T. Functional brain mapping of extraversion and neuroticism: learning from individual differences in emotion processing. Journal of personality 72, 1105-32 (2004).

4. Canli, T., Sivers, H., Whitfield, S. L., Gotlib, I. H. \& Gabrieli, J. D. Amygdala response to happy faces as a function of extraversion. science 296, 2191 (2002).

5. Cremers, H. et al. Extraversion is linked to volume of the orbitofrontal cortex and amygdala. PloS one 6, e28421 (2011).

6. Cremers, H. R. et al. Neuroticism modulates amygdala-prefrontal connectivity in response to negative emotional facial expressions. Neuroimage 49, 963-70 (2010).

7. Dima, D., Friston, K. J., Stephan, K. E. \& Frangou, S. Neuroticism and conscientiousness respectively constrain and facilitate shortterm plasticity within the working memory neural network. Hum Brain Mapp 36, 4158-63 (2015).

8. Fischer, H., Wik, G. \& Fredrikson, M. Extraversion, neuroticism and brain function: A PET study of personality. Personality and Individual Differences 23, 345-352 (1997).

9. Hu, X. et al. Voxel-based morphometry studies of personality: issue of statistical model specification-effect of nuisance covariates. Neuroimage 54, 1994-2005 (2011).

10. Kapogiannis, D., Sutin, A., Davatzikos, C., Costa, P. \& Resnick, S. The five factors of personality and regional cortical variability in the baltimore longitudinal study of aging. Hum Brain Mapp, https://doi.org/10.1002/hbm.22108 (2012).

11. Krebs, R. M., Schott, B. H. \& Duzel, E. Personality traits are differentially associated with patterns of reward and novelty processing in the human substantia nigra/ventral tegmental area. Biol Psychiatry 65, 103-10 (2009).

12. Liu, W. Y. et al. The Big Five of Personality and structural imaging revisited: a VBM - DARTEL study. Neuroreport 24, 375-80 (2013).

13. Lu, F. et al. Relationship between personality and gray matter volume in healthy young adults: a voxel-based morphometric study. PloS one 9, e88763 (2014).

14. Passamonti, L. et al. Increased functional connectivity within mesocortical networks in open people. Neuroimage 104, 301-9 (2015).

15. Rodrigo, A. H. et al. Linking trait-based phenotypes to prefrontal cortex activation during inhibitory control. Soc Cogn Affect Neurosci 11, 55-65 (2016).

16. Servaas, M. N. et al. Connectomics and neuroticism: an altered functional network organization. Neuropsychopharmacology $\mathbf{4 0}$, 296-304 (2015).

17. Wright, C. I. et al. Neuroanatomical correlates of extraversion and neuroticism. Cerebral cortex 16, 1809-19 (2006).

18. Indovina, I., Riccelli, R., Staab, J. P., Lacquaniti, F. \& Passamonti, L. Personality traits modulate subcortical and cortical vestibular and anxiety responses to sound-evoked otolithic receptor stimulation. J Psychosom Res 77, 391-400 (2014).

19. Riccelli, R. et al. Neuroticism modulates brain visuo-vestibular and anxiety systems during a virtual rollercoaster task. Hum Brain Mapp 38, 715-726 (2017)

20. Berger, P., Bitsch, F., Nagels, A., Straube, B. \& Falkenberg, I. Personality modulates amygdala and insula connectivity during humor appreciation: An event-related fMRI study. Soc Neurosci 1-13, https://doi.org/10.1080/17470919.2017.1403375 (2017).

21. Angelides, N. H., Gupta, J. \& Vickery, T. J. Associating resting-state connectivity with trait impulsivity. Soc Cogn Affect Neurosci 12, 1001-1008 (2017).

22. Deris, N., Montag, C., Reuter, M., Weber, B. \& Markett, S. Functional connectivity in the resting brain as biological correlate of the Affective Neuroscience Personality Scales. Neuroimage 147, 423-431 (2017).

23. Markett, S., Montag, C., Melchers, M., Weber, B. \& Reuter, M. Anxious personality and functional efficiency of the insular-opercular network: A graph-analytic approach to resting-state fMRI. Cogn Affect Behav Neurosci 16, 1039-1049 (2016).

24. Pang, Y. et al. Extraversion modulates functional connectivity hubs of resting-state brain networks. J Neuropsychol 11, 347-361 (2017).

25. Toschi, N., Riccelli, R., Indovina, I., Terracciano, A. \& Passamonti, L. Functional Connectome of the Five-Factor Model of Personality. Personality Neuroscience 1 (2018).

26. Vidaurre, D., Smith, S. M. \& Woolrich, M. W. Brain network dynamics are hierarchically organized in time. Proc Natl Acad Sci USA, https://doi.org/10.1073/pnas.1705120114 (2017).

27. Zalesky, A., Fornito, A., Cocchi, L., Gollo, L. L. \& Breakspear, M. Time-resolved resting-state brain networks. Proc Natl Acad Sci USA 111, 10341-6 (2014).

28. Hindriks, R. et al. Can sliding-window correlations reveal dynamic functional connectivity in resting-state fMRI? Neuroimage 127, 242-256 (2016)

29. Hutchison, R. M. et al. Dynamic functional connectivity: promise, issues, and interpretations. Neuroimage 80, 360-78 (2013).

30. Robinson, L. F. et al. The Temporal Instability of Resting State Network Connectivity in Intractable Epilepsy. Hum Brain Mapp 38, 528-540 (2017) 
31. Hindriks, R. et al. Corrigendum to 'Can sliding-window correlations reveal dynamic functional connectivity in resting-state fMRI?' [NeuroImage 127 (2016) 242-256]. Neuroimage 132, 115 (2016).

32. Lindquist, M. A., Xu, Y., Nebel, M. B. \& Caffo, B. S. Evaluating dynamic bivariate correlations in resting-state fMRI: a comparison study and a new approach. Neuroimage 101, 531-46 (2014).

33. Costa, P. T. \& McCrae, R. R. Revised NEO Personality Inventory (NEO-PI-R) and NEO Five-Factor Inventory (NEO-FFI) professional manual. (Odessa, FL: Psychological Assessment Resources, 1992).

34. Digman, J. M. Personality structure: Emergence of the five-factor model. Annual review of psychology 41, 417-440 (1990).

35. McCrae, R. R. \& Terracciano, A. Universal features of personality traits from the observer's perspective: data from 50 cultures. Journal of personality and social psychology 88, 547 (2005).

36. Terracciano, A., An, Y., Sutin, A. R., Thambisetty, M. \& Resnick, S. M. Personality Change in the Preclinical Phase of Alzheimer Disease. JAMA. Psychiatry. https://doi.org/10.1001/jamapsychiatry.2017.2816 (2017).

37. Kern, M. L. \& Friedman, H. S. Do conscientious individuals live longer? A quantitative review. Health Psychol 27, 505-12 (2008).

38. Sutin, A. R., Luchetti, M., Stephan, Y., Robins, R. W. \& Terracciano, A. Parental educational attainment and adult offspring personality: An intergenerational life span approach to the origin of adult personality traits. J Pers Soc Psychol 113, 144-166 (2017).

39. Raichle, M. E. The restless brain: how intrinsic activity organizes brain function. Philos Trans R Soc Lond B Biol Sci 370 (2015).

40. Toschi, N., Duggento, A. \& Passamonti, L. Functional connectivity in amygdalar-sensory/(pre)motor networks at rest: new evidence from the Human Connectome Project. Eur. J. Neurosci. 45, 1224-1229 (2017).

41. Despotovic, M., Nedic, V., Despotovic, D. \& Cvetanovic, S. Evaluation of empirical models for predicting monthly mean horizontal diffuse solar radiation. Renewable and Sustainable Energy Reviews 56, 246-260 (2016).

42. Ozer, D. J. \& Benet-Martinez, V. Personality and the prediction of consequential outcomes. Annu Rev Psychol 57, 401-21 (2006).

43. Roberts, B. W., Lejuez, C., Krueger, R. F., Richards, J. M. \& Hill, P. L. What is conscientiousness and how can it be assessed? Dev Psychol 50, 1315-30 (2014).

44. Noftle, E. E. \& Robins, R. W. Personality predictors of academic outcomes: big five correlates of GPA and SAT scores. J Pers Soc Psychol 93, 116-30 (2007).

45. Sutin, A. R. et al. The five-factor model of personality and physical inactivity: A meta-analysis of 16 samples. Journal of Research in Personality 63, 22-28 (2016).

46. DeYoung, C. G. Cybernetic Big Five Theory. Journal of Research in Personality 56, 33-58 (2015).

47. Donnellan, M. B., Conger, R. D. \& Burzette, R. G. Personality development from late adolescence to young adulthood: Differential stability, normative maturity, and evidence for the maturity-stability hypothesis. Journal of personality 75, 237-264 (2007).

48. Lönnqvist, J.-E., Mäkinen, S., Paunonen, S. V., Henriksson, M. \& Verkasalo, M. Psychosocial functioning in young men predicts their personality stability over 15 years. Journal of Research in Personality 42, 599-621 (2008).

49. Blonigen, D. M., Carlson, M. D., Hicks, B. M., Krueger, R. F. \& Iacono, W. G. Stability and change in personality traits from late adolescence to early adulthood: A longitudinal twin study. Journal of personality 76, 229-266 (2008).

50. Terracciano, A., Stephan, Y., Luchetti, M. \& Sutin, A. R. Cognitive Impairment, Dementia, and Personality Stability Among Older Adults. Assessment 25, 336-347 (2018)

51. Roberts, B. W., Caspi, A. \& Moffitt, T. E. The kids are alright: growth and stability in personality development from adolescence to adulthood. Journal of personality and social psychology 81, 670 (2001).

52. Eisenberg, N., Duckworth, A. L., Spinrad, T. L. \& Valiente, C. Conscientiousness: origins in childhood? Dev Psychol 50, 1331-49 (2014).

53. DeYoung, C. G. et al. Testing predictions from personality neuroscience. Brain structure and the big five. Psychol Sci 21, 820-8 (2010).

54. Arana, J. M., Meilan, J. J. \& Perez, E. The effect of personality variables in the prediction of the execution of different prospective memory tasks in the laboratory. Scand J Psychol 49, 403-11 (2008).

55. Cuttler, C. \& Graf, P. Personality predicts prospective memory task performance: an adult lifespan study. Scand J Psychol 48, 215-31 (2007).

56. Smith, R. E., Persyn, D. \& Butler, P. Prospective Memory, Personality, and Working Memory: A Formal Modeling Approach. Z Psychol 219, 108-116 (2011).

57. Salimi-Khorshidi, G. et al. Automatic denoising of functional MRI data: combining independent component analysis and hierarchical fusion of classifiers. Neuroimage 90, 449-68 (2014).

58. Smith, S. M. et al. Resting-state fMRI in the Human Connectome Project. Neuroimage 80, 144-68 (2013)

59. Griffanti, L. et al. ICA-based artefact removal and accelerated fMRI acquisition for improved resting state network imaging. Neuroimage 95, 232-47 (2014).

60. Terracciano, A. The Italian version of the NEO PI-R: conceptual and empirical support for the use of targeted rotation. Personality and individual differences 35, 1859-1872 (2003).

61. Van Essen, D. C. et al. The Human Connectome Project: a data acquisition perspective. Neuroimage 62, 2222-2231 (2012).

62. Glasser, M. F. et al. The minimal preprocessing pipelines for the Human Connectome Project. Neuroimage 80, 105-124 (2013).

63. Smith, S. M., Hyvärinen, A., Varoquaux, G., Miller, K. L. \& Beckmann, C. F. Group-PCA for very large fMRI datasets. Neuroimage 101, 738-749 (2014).

64. Beckmann, C. F. \& Smith, S. M. Probabilistic independent component analysis for functional magnetic resonance imaging. IEEE Trans Med Imaging 23, 137-52 (2004).

65. Engle, R. Dynamic Conditional Correlation. Journal of Business \& Economic Statistics 20, 339-350 (2002).

\section{Acknowledgements}

Roberta Riccelli is funded by the University "Tor Vergata” of Rome, Italy, while Luca Passamonti is funded by the Medical Research Council (MRC) (MR/P01271X/1) at the University of Cambridge, UK. Antonio Terracciano is supported by the National Institute on Aging of the National Institutes of Health under Award Number R01AG053297 and R03AG051960. Iole Indovina is funded by the Italian Ministry of Health (PE-2013-02355372). Data collection and sharing for this project was provided by the MGH-USC Human Connectome Project (HCP; Principal Investigators: Bruce Rosen, M.D., Ph.D., Arthur W. Toga, Ph.D., Van J. Weeden, MD). The HCP project is supported by the National Institute of Dental and Craniofacial Research (NIDCR), the National Institute of Mental Health (NIMH) and the National Institute of Neurological Disorders and Stroke (NINDS) (Principal Investigators: Bruce Rosen, M.D., Ph.D., Martinos Center at Massachusetts General Hospital; Arthur W. Toga, Ph.D., University of Southern California, Van J. Weeden, MD, Martinos Center at Massachusetts General Hospital). 


\section{Author contributions}

L.P. helped conceptualize and design the study, interpreted the results, drafted the manuscript, critically reviewed and revised the manuscript, and approved the final manuscript as submitted. R.R. led the data analysis, critically reviewed and revised the manuscript, and approved the final manuscript as submitted. I.I. provided methodological perspective on the study, critically reviewed the initial manuscript and approved the final manuscript as submitted. A.D. provided methodological perspective on the study, designed the figures and critically reviewed the initial manuscript. A.T. helped to conceptualize the study, critically reviewed the initial manuscript and approved the final manuscript as submitted. N.T. conceptualized, designed, and supervised all aspects of the study, including data analysis, and approved the final manuscript as submitted.

\section{Competing interests}

The authors declare no competing interests.

\section{Additional information}

Supplementary information is available for this paper at https://doi.org/10.1038/s41598-019-51469-2.

Correspondence and requests for materials should be addressed to L.P.

Reprints and permissions information is available at www.nature.com/reprints.

Publisher's note Springer Nature remains neutral with regard to jurisdictional claims in published maps and institutional affiliations.

(c) (i) Open Access This article is licensed under a Creative Commons Attribution 4.0 International License, which permits use, sharing, adaptation, distribution and reproduction in any medium or format, as long as you give appropriate credit to the original author(s) and the source, provide a link to the Creative Commons license, and indicate if changes were made. The images or other third party material in this article are included in the article's Creative Commons license, unless indicated otherwise in a credit line to the material. If material is not included in the article's Creative Commons license and your intended use is not permitted by statutory regulation or exceeds the permitted use, you will need to obtain permission directly from the copyright holder. To view a copy of this license, visit http://creativecommons.org/licenses/by/4.0/.

(C) The Author(s) 2019 